\title{
Weak interaction and matching conditions for replicas of vortex lattices
}

\author{
Yanina Fasano, M. Menghini, F. de la Cruz, and G. Nieva \\ Centro Atómico Bariloche and Instituto Balseiro, CNEA-UNC, 8400 San Carlos de Bariloche, RN, Argentina
}

(Received 25 April 2000)

\begin{abstract}
We present experiments demonstrating the conditions under which weak interaction between replicas of vortex structures break their translational and rotational symmetry. The Fe clumps introduced by Bitter decorations of vortex structures are used to induce an extremely weak interaction for subsequent vortex structures in low and high temperature superconducting crystals. The matching between the Fe pattern and the vortex configuration require the structure to be prepared under quite stringent conditions: The field creating the structure has to be the same in magnitude although it can differ in orientation from that used for the preparation of the Fe pattern. The interaction between vortex replicas as represented by the Fe clumps of Bitter decorations makes it possible to differentiate the vortex nucleation and propagation mechanisms in low and high temperature superconductors.
\end{abstract}

\section{INTRODUCTION}

The need for understanding a wide class of phenomena in the high critical temperature $\left(T_{C}\right)$ superconductors promoted the reconsideration of a variety of previously accepted arguments in conventional superconductors. ${ }^{1}$ Among those, the role played by pinning potentials (disordered as well as periodic) and thermally induced disorder have triggered theoretical and experimental work. ${ }^{2-8}$

Several techniques have been developed in recent years to artificially create pinning centers for studying the relevance of the intensity, topology and morphology of the potential in the thermodynamic and dynamic properties of the vortex structures. $^{5-8}$ These technologies have been extensively used to analyze the behavior of vortex lattices interacting either with commensurate or incommensurate pinning structures. In the former case magnetic dots arranged in a periodic structure in low temperature superconducting films are one of the most successful approaches.

Transport measurements verify that the pinning induced by periodic magnetic dots overwhelms that of the bulk, whenever the flux induced in the unit cell of the pinning structure is a multiple of the flux quantum. ${ }^{6}$ On the other hand, the lattice parameter of the periodic pinning pattern is large when compared with typical lengths determining the Larkin pinning volume of the films studied. As a consequence, the phase space that the vortices are required to span to match the artificial pinning is much larger than allowed by reasonable relaxation times in typical strong pinning films.

The apparent inconsistency between the results of transport measurements (showing commensurability between the vortex-pinning structures) and the small Larkin volumes in films (suggesting a random distribution of vortices) can be solved assuming that the matching between structures is a consequence of the ordering induced by vortex dynamics. ${ }^{9}$ To detect dissipation in transport measurements current densities must exceed the critical one. Thus, long range dynamical ordering might be induced in the vortex structure as detected by neutron ${ }^{10}$ and decoration experiments. ${ }^{11,12}$ In this picture, the periodic response is not associated with a peri- odic critical current resulting from a static match of the vortex and pinning patterns.

To verify the above scenario it is important to detect the position of vortices in static and dynamic experiments to obtain information on how the vortex structure nucleates and propagates. Bitter decoration is an appropriate technique for this purpose. However, it is important to understand how the decoration made at low temperatures provides information on the nucleation mechanism of the vortex structures at higher temperatures.

(a) When a low- $T_{C}$ superconductor is decorated in a field cooling (FC) experiment the image of Fe clumps corresponds to a frozen structure that closely resembles the one at the vicinity of $H_{C 2}(T)$, where vortices are nucleated and pinned at the same time. When the bulk pinning associated with randomly distributed pinning centers is in the strong limit they become nucleation centers simultaneously. As a result, the vortex pattern is nucleated at the second order superconducting phase transition in a highly disordered or even amorphous pattern. ${ }^{13}$ On the other hand if pinning is in the weak limit, nucleation and growth of vortex crystalline grains in different directions take place. The decoration shows a frozen polycrystalline structure where most of the defects are determined by grain boundaries.

(b) In a high- $T_{C}$ superconductor a liquid vortex state is first formed when decreasing temperature from above the critical. The FC decoration represents the frozen vortex structure at some temperature below the melting transition. ${ }^{13}$ Therefore, if the solid structure is achieved through a first order freezing, the decoration should show a crystalline structure independently of the presence of pinning sites. This is because at the melting temperature, where the solid structure is formed, the vortex-vortex interaction is stronger than that producing vortex pinning. As temperature decreases, pinning becomes stronger and the vortex pattern is frozen. This process is accomplished by small displacements of every vortex within the range of the pinning potential (much smaller than the vortex lattice parameter) and consequently the overall crystalline order is preserved.

In this work we focus the attention on the real space observation of static and dynamic vortex structures responding 
to the presence of quasiperiodic and disordered pinning potentials. We investigate whether the vortex pattern in the sample is the result of the structure growth from nucleation centers distributed at random or if it follows a predetermined pattern characteristic of each sample. This is achieved using a simple technique recently developed in our laboratory that allows us to engineer pinning potentials by means of a novel method: Bitter pinning. In this technique the Fe clumps used to observe the vortex structure in a first Bitter decoration become pinning centers for new vortex structures. A second decoration allows the observation of the new vortex pattern and the comparison with the Fe clump distribution of the first one.

The Bitter pinning technique is shown to be appropriate to determine the conditions for an effective matching between vortex and artificially induced pinning structures. We demonstrate that the matching condition is rather subtle and does not require the expected stronger pinning associated with the Fe pattern, as compared with that of the bulk. When bulk pinning is essentially zero an extremely weak quasiperiodic potential is capable of locking commensurate vortex structures breaking the translational as well as the rotational symmetry of the vortex lattice.

\section{EXPERIMENTAL TECHNIQUE AND SAMPLE CHARACTERIZATION}

In the vortex decoration technique small $\mathrm{Fe}$ particles (of the order of $200 \AA$ diameter) diffusing in a He gas atmosphere are attracted by the field modulation produced by the vortex pattern at the surface of the sample. ${ }^{14}$ The clumps formed by the iron particles remain attached to the surface by van der Waals forces and are observed by SEM at room temperature. In the Bitter pinning the sample is cooled again in the presence of the Fe clumps and the effect of the surface introduced pinning on the new vortex structure is analyzed.

In all the experiments described in this work the vortices are nucleated in field cooling experiments.

The influence of the pinning induced by the Fe clumps on the critical current is studied by dc electrical resistance, magnetization loops and ac susceptibility measurements.

The relevance of the resultant weak $\mathrm{Fe}$ periodic pinning potential on the vortex distribution and correlation in real space is made evident by the following procedure: Once the sample is decorated in a first FC experiment it is warmed up to room temperature to observe the Fe clumps. Subsequently, the sample is $\mathrm{FC}$ again down to $4 \mathrm{~K}$ and a second Bitter decoration is performed to observe the vortex structure. A portion of the sample is masked in the first decoration in order to verify the quality of the second one. Details of the technique have been reported elsewhere. ${ }^{15,16}$

The degree of periodic order in the first Fe pattern to be used in the Bitter pinning can be controlled by making use of two effects: dynamical ordering and dynamical memory.

Dynamical ordering was theoretically predicted and observed experimentally and in computer simulations. ${ }^{9,17-19}$ It has been shown that the disorder of vortex patterns nucleated in the presence of random potentials is strongly reduced when the vortex structure is displaced by forces exceeding the critical.

Dynamical memory is an experimental result showing

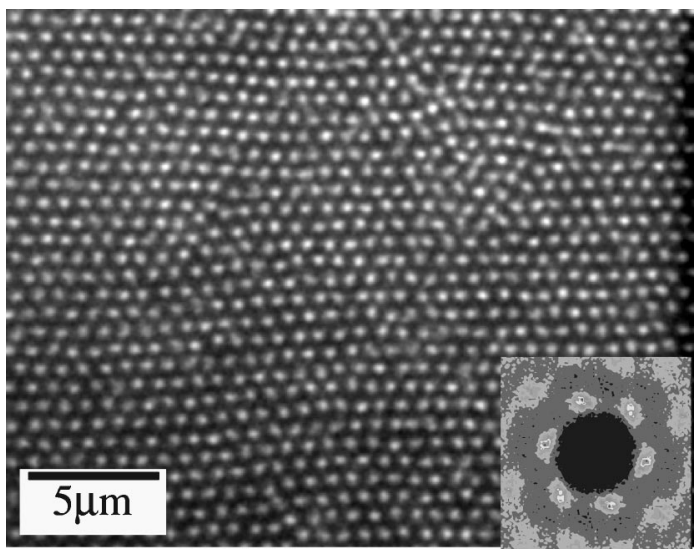

FIG. 1. Magnetic decoration pattern of the flux-line lattice in $\mathrm{NbSe}_{2}$ superconductor. The white dots depict the positions of the vortices after a FCR process (see text) down to $4.2 \mathrm{~K}$ at 36 Oe with a field rotation at an angle of $60^{\circ}$. In the inset, the Fourier transform of the vortex pattern shows a six peak structure that makes evident its crystalline nature.

that the dynamically induced order is preserved when the force acting on the vortex structure is suddenly removed. This memory effect shows that the direction of the applied force determines the orientation of the vortex quenched lattice. Dynamical memory is of basic importance to produce the oriented quasiperiodic pinning potential of the Bitter pinning technique. In this way polycrystalline vortex structures nucleated in $\mathrm{NbSe}_{2}$ are transformed into almost perfect periodic structures extended over the whole sample. ${ }^{15}$ An example is shown in Fig. 1: Thousands of Fe dots induced in the first decoration become the quasiperiodic structure to be used in subsequent decorations.

A simple technique to generate dynamical ordering and preserve memory is to induce currents exceeding the critical by rapid magnetic field rotations. ${ }^{20}$ This method has the advantage of inducing currents with no need of electrical contacts in the sample. The sample is FC from room temperature down to $4 \mathrm{~K}$, the applied magnetic field is rotated to a given angle and then rapidly returned to the original orientation. The rotation used in the experiments described in this paper is accomplished by switching on and off a field $H_{T}$, perpendicular to the applied one. We call this experiment field cooling rotation (FCR).

The samples used in this work are single crystals of $\mathrm{Bi}_{2} \mathrm{Sr}_{2} \mathrm{CaCu}_{2} \mathrm{O}_{8}$ grown using the self-flux technique in a large temperature gradient. ${ }^{21}$ The samples are annealed in an Ar atmosphere at $500{ }^{\circ} \mathrm{C}$ for $20 \mathrm{~h}$ followed by a fast cool down to achieve optimal doping. The crystals are characterized by x-ray diffraction and energy dispersive spectroscopy (EDS) to ensure the right structure and composition. The critical temperature $T_{C} \approx 87 \mathrm{~K}$ is determined using ac susceptibility measurements. The superconducting parameters are $\lambda \approx 2000 \AA$ and $\xi \approx 10 \AA$. Typical sample dimensions are 1 $\mathrm{mm}^{2}$ area and $50 \mu \mathrm{m} 1$ thickness, with the $c$ axis oriented parallel to the thin dimension.

In order to keep the surface of the sample in optimal conditions the decoration is made using different freshly cleaved samples. 


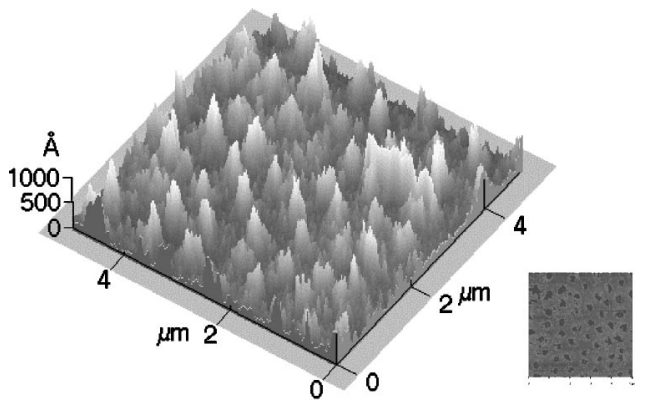

FIG. 2. AFM topographic characteristics of the iron structure generated by a magnetic decoration. The three-dimensional image depicts cone shaped iron clumps with an average height of $600 \AA$ and average base diameter of $2000 \AA$.

\section{PINNING INDUCED BY IRON CLUMPS}

The pinning induced by the Fe clumps is extremely weak. Its effect on the dynamics of the vortex state is undetectable by dc resistance, ac susceptibility and magnetization loop measurements. This is understood by considering that the ratio between the length of the vortex (thickness of the sample) and the height of the Fe clumps on the surface is in the range between 200 and 400 for the samples used.

The shape and dimensions of the Fe dots after the first and second decorations are determined by means of an AFM used in the constant force mode. Typical results are shown in Fig. 2 for a first decoration in a $\mathrm{Bi}_{2} \mathrm{Sr}_{2} \mathrm{CaCu}_{2} \mathrm{O}_{8}$ sample at 36 Oe. The Fe clumps of conical shape have an average height of $600 \pm 20 \AA$ and a base diameter of $2000 \pm 100 \AA$. This low dispersion in the dimensions of the Fe clumps implies homogeneity of the Bitter pinning potential in the whole sample.

\section{ANALYSIS OF PREVIOUS RESULTS IN $\mathrm{NbSe}_{2}$}

In order to provide an adequate frame for the analysis of the results in $\mathrm{Bi}_{2} \mathrm{Sr}_{2} \mathrm{CaCu}_{2} \mathrm{O}_{8}$ it is important to make a brief review and a discussion of previous results ${ }^{15}$ in the low temperature superconductor $\mathrm{NbSe}_{2}$.

The FC magnetic decoration in $\mathrm{NbSe}_{2}$ crystals shows a polycrystalline vortex structure as expected for high quality low temperature superconducting material.

It has been shown previously that applying the Bitter pinning technique to the polycrystalline vortex structure, matching is only found between grains of both decorations that grow in the same direction. ${ }^{15,16}$ Thus, no relative translation between structures have been found from the observation of many decorations. On the other hand, when the direction of growth of the second structure differs from that of the first decoration the crystal is only partially pinned by the Bitter pinning, as shown by the Moiré patterns. ${ }^{15,16}$ Typical examples of Bitter pinning showing the configuration mentioned before are depicted in Fig. 3.

The results in Fig. 3 shed light on the mechanism of crystal domain growth in $\mathrm{NbSe}_{2}$. Figure 3(a) indicates that the $\mathrm{Fe}$ structure in the Bitter pinning breaks the translational symmetry of a second commensurate pattern with the same lattice parameter when growing in a direction parallel to the $\mathrm{Fe}$ structure. That is, from the many possible pinned vortex configurations (within the range of distances determined by the
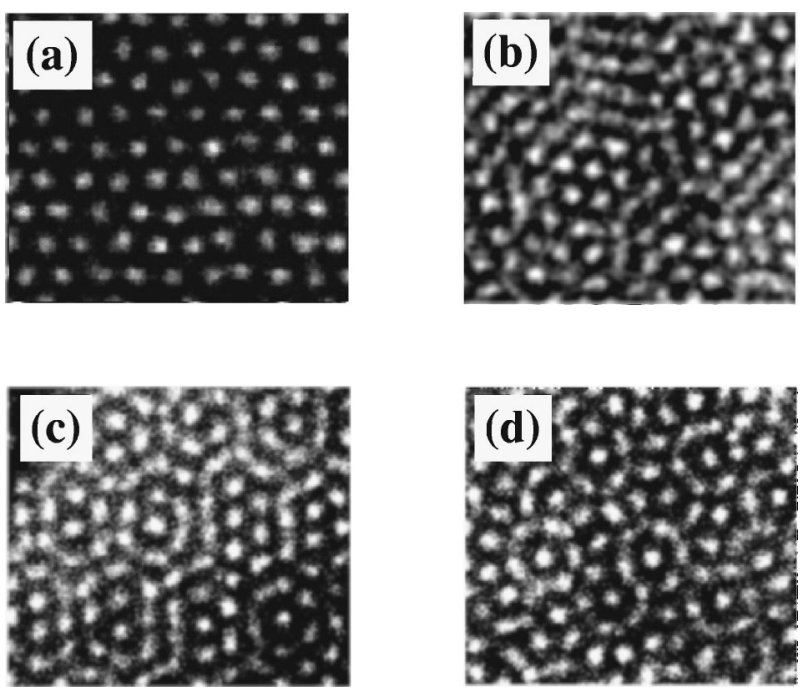

FIG. 3. Decoration pattern obtained in $\mathrm{NbSe}_{2}$ in a FC Bitter pinning experiment. The iron pinning structure is generated by a first vortex decoration in a FC experiment at 36 Oe and $4.2 \mathrm{~K}$. Subsequently, the sample is warmed up to room temperature and then FC again at the same field and down to the same temperature in the presence of the additional Bitter pinning potential. Then, the second decoration is performed to analyze the vortex positions of the structure with respect to the iron pinning clumps. Different patterns in the sample show local rotations between the second vortex lattice and the Bitter pinning. Examples of Moiré patterns corresponding to different rotation angles are shown in the figure: (a) $0^{\circ}$, (b) $10^{\circ}$, (c) $40^{\circ}$, and (d) $30^{\circ}$.

range of the bulk pinning potential) there is one with the lowest energy determined by the Fe clumps. On the other hand, the Moiré patterns in Figs. 3(b), 3(c), and 3(d) prove that the Fe clumps are not capable of breaking the rotational degeneracy of crystal growth. The data strongly support that the nucleation and growth direction of the vortex lattice in $\mathrm{NbSe}_{2}$ is at random and induced by bulk pinning. Thus, the periodic Fe superficial pinning is ineffective for determining the propagation direction of the nucleated vortex lattice.

By means of the dynamical memory technique the relative importance of the bulk pinning is suppressed or strongly diminished. Consequently, structures with long range order grow in the direction determined by the force applied on the vortex lattice. In this case, the Bitter pinning technique at the same field shows perfect matching between the two structures. As it will be discussed later, the Fe structure breaks the translational and rotational degeneracy of a lattice when quenched from a dynamical state induced by forces exceeding the critical. An example can be observed in Fig. 4, where dynamical ordering was induced by the rapid field rotation method with an angle of $60^{\circ}$. It is interesting to remark that in this case the dislocations present in the first decoration are reproduced in the second one. If dislocations of the two decorations were not correlated the Bitter technique would induce a new plane of $\mathrm{Fe}$ dots and an apparent plane of defects after the second decoration would appear. This has never been observed.

\section{RESULTS AND DISCUSSION IN $\mathrm{Bi}_{2} \mathrm{Sr}_{2} \mathrm{CaCu}_{2} \mathrm{O}_{8}$}

The high temperature superconductor $\mathrm{Bi}_{2} \mathrm{Sr}_{2} \mathrm{CaCu}_{2} \mathrm{O}_{8}$ has a rich $H-T$ phase diagram ${ }^{22}$ complementary to that of $\mathrm{NbSe}_{2}$ 

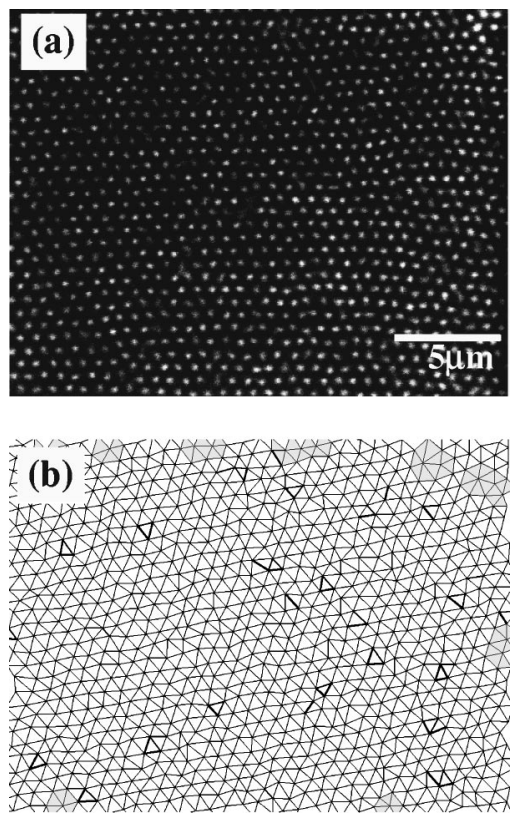

FIG. 4. Decoration pattern obtained in $\mathrm{NbSe}_{2}$ in a FCR Bitter pinning experiment (see text). The iron pinning structure is generated by a FCR first vortex decoration in 36 Oe with a 60 degrees rotation at $4.2 \mathrm{~K}$. Subsequently, a second FCR vortex decoration under the same field and temperature conditions is performed in the presence of the additional Bitter pinning potential. The picture shows perfect matching between the two dynamically ordered vortex lattices. (a) Double magnetic decoration in real space. (b) Delaunay triangulation of the structure where even the topological defects of the Bitter pinning pattern are shown to be reproduced in the second decoration.

for the study of vortex nucleation and propagation. When FC the sample below $T_{C}$ a vortex liquid phase transforms into an ordered crystalline solid through a first order phase transition. $^{23}$

Field cooling decorations at $4 \mathrm{~K}$ in a field of 36 Oe make evident the high quality of the vortex crystals; see Fig. 5.

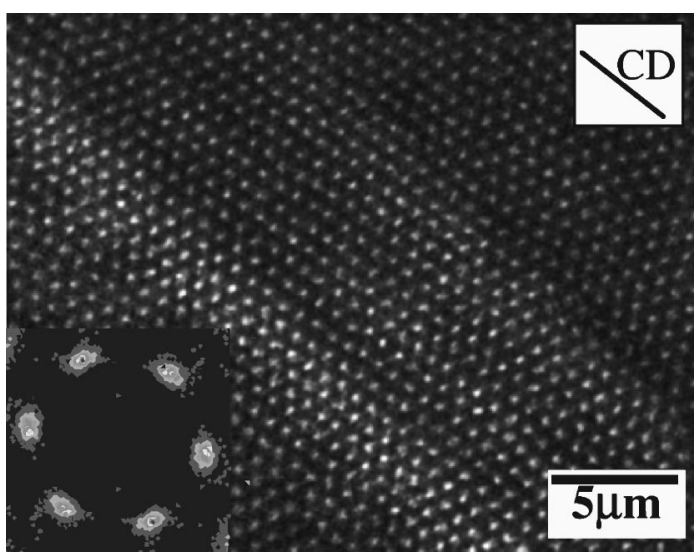

FIG. 5. Vortex lattice in $\mathrm{Bi}_{2} \mathrm{Sr}_{2} \mathrm{CaCu}_{2} \mathrm{O}_{8}$ in a $\mathrm{FC}$ experiment in $36 \mathrm{Oe}$ at $4.2 \mathrm{~K}$ obtained with magnetic decoration. One of the principal directions of the hexagonal vortex lattice is shown to coincide with the direction of the correlated defects (CD) present in other regions of the sample. The Fourier transform of the vortice positions shown in the inset makes evident the hexagonal symmetry of the vortex lattice.
Contrary to what is observed in $\mathrm{NbSe}_{2}$ there is no need of Dynamical Ordering to induce long range order, as shown by the Fourier transform in the insert of the figure.

The density of topological defects in the vortex structure is the same as that observed in FCR experiments in $\mathrm{NbSe}_{2}$ at the same field, despite the fact that the critical current of the $\mathrm{Bi}_{2} \mathrm{Sr}_{2} \mathrm{CaCu}_{2} \mathrm{O}_{8}$ crystals at $4 \mathrm{~K}$ is more than two orders of magnitude larger. This result supports the mechanism described in the introduction for explaining the coexistence of strong pinning at low temperatures and the preservation of the ordered vortex structure nucleated at higher temperatures. The absence of grain boundaries seen in most pictures of the vortex structure in $\mathrm{Bi}_{2} \mathrm{Sr}_{2} \mathrm{CaCu}_{2} \mathrm{O}_{8}$ suggests a different nucleation and growth process of the vortex pattern. This finding triggered our interest to discover the nature of nucleation centers from which crystalline order propagates.

Studying the vortex decoration in extended regions of the sample it is found that even the best samples of $\mathrm{Bi}_{2} \mathrm{Sr}_{2} \mathrm{CaCu}_{2} \mathrm{O}_{8}$ have correlated atomic defects of unknown origin, see Figs. 6(d) and 6(e). These defects are not detected by standard material characterization techniques such as SEM pictures, EDS, XR. The vortices in the region of correlated defects nucleate in a disorderly way along lines with higher density $(\approx 4 \%$ ) than that in the region free of defects. No correlation between vortices in different lines is found. The defects detected by the Bitter decoration with single vortex resolution are quite likely the same as those found with magneto-optical techniques. ${ }^{24}$

Two remarkable characteristics of the $\mathrm{Bi}_{2} \mathrm{Sr}_{2} \mathrm{CaCu}_{2} \mathrm{O}_{8}$ single crystals are to a large extent determinant of the properties of the vortex structure in the range of fields and temperatures where the decoration is made: The presence of correlated defects in some regions of the sample and the very weak or absent bulk vortex pinning at the melting temperature. As a consequence high quality vortex crystals are obtained.

It is remarkable that the transition from vortex disorder in the region of correlated defects to the crystalline structure is produced at a sharp boundary defined with precision of one lattice constant. Correlated defects impose the growth direction of the vortex crystal as seen in Fig. 6(d) and 6(e), where a compact plane of the hexagonal vortex crystalline structure is shown to be parallel to the defects.

Surface steps is the other detected vortex pinning potential that competes with correlated defects to establish the growth direction of the vortex crystal. In Figs. 6(a), 6(b), and $6(c)$ the compact plane of the hexagonal structure is seen to grow parallel to the surface step shown in the picture. The occasional grain boundaries found in the $\mathrm{Bi}_{2} \mathrm{Sr}_{2} \mathrm{CaCu}_{2} \mathrm{O}_{8}$ vortex patterns are induced by the intersection of crystalline structures nucleated and oriented by both kinds of defects; see Fig. 7. Starting from the grain boundary it is easy to track the correlated defects or surface step that produce the different orientations; see Figs. 6 and 7.

The Bitter pinning in $\mathrm{Bi}_{2} \mathrm{Sr}_{2} \mathrm{CaCu}_{2} \mathrm{O}_{8}$ induces perfect matching of two lattices with the same lattice parameter, with no need of previous dynamical ordering. In agreement with this, AFM measurements of the Fe clumps height after a second decoration in the crystalline region is on average twice that of the first decoration $(1200 \pm 200 \AA)$. The extremely weak superficial pinning potential breaks the trans- 


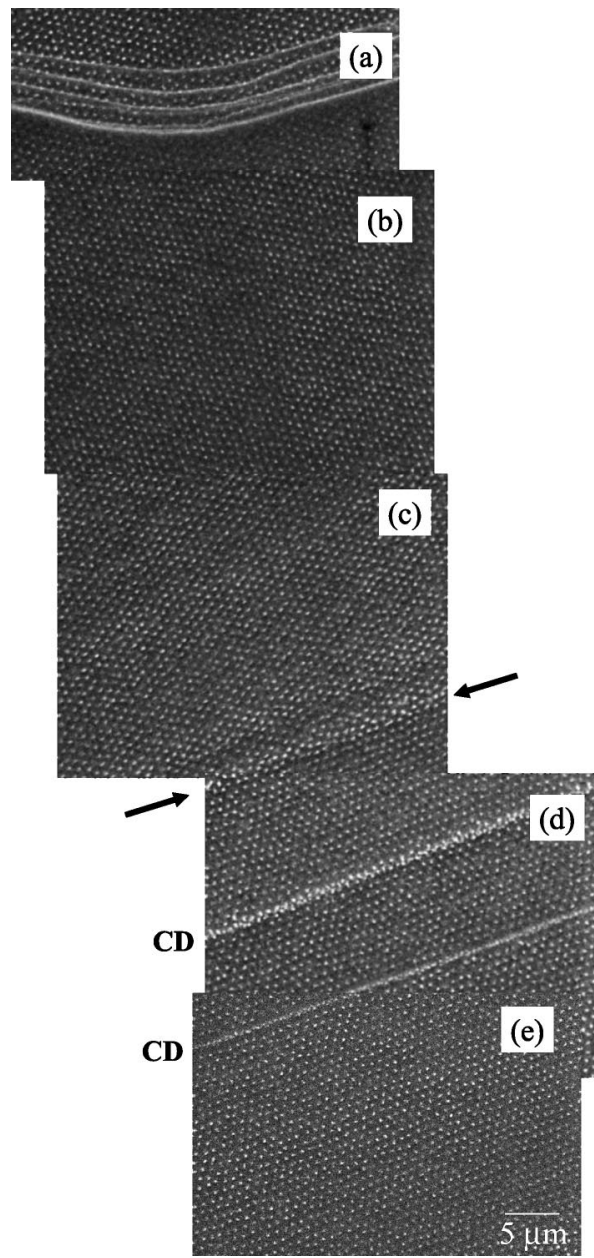

FIG. 6. Sequence of several pictures showing vortex positions in $\mathrm{Bi}_{2} \mathrm{Sr}_{2} \mathrm{CaCu}_{2} \mathrm{O}_{8}$ after a $\mathrm{FC}$ experiment in $36 \mathrm{Oe}$ as determined by magnetic decoration at $4.2 \mathrm{~K}$ in an extended region of the sample. (a) Surface step determining the growing direction of the vortex lattice. (b) Continuation of the crystalline vortex lattice shown in (a). (c) Grain boundary (see arrows) resulting from the intersection of the lattice shown in (a) and (b) with the vortex lattice oriented in a direction determined by correlated defects (CD) shown in (d) and (e).

lational degeneracy expected for nucleation and growth of the vortex structure. The results of the decoration support the suggestion of null or very weak bulk pinning potential when the crystalline structure is nucleated from the liquid state.

The second decoration in the region with correlated defects does not reproduce the disordered pattern of the Fe particles of the first one. That is, the Fe pinning becomes ineffective to pin disordered structures.

It is important to emphasize that the Bitter pinning induced with $H=36$ Oe acting on the periodic vortex structure of $\mathrm{Bi}_{2} \mathrm{Sr}_{2} \mathrm{CaCu}_{2} \mathrm{O}_{8}$ (with no dynamically induced order) is as effective as it is in $\mathrm{NbSe}_{2}$ after dynamically ordering the first as well as the second structure with the same applied field. Thus, in both cases the Fe replica of the first decoration establishes a space configuration that lowers the free energy of a second identical vortex structure. The matching in the second decoration implies that the dislocations of the first structure are reproduced at the same sample sites; see Fig. 8 (a) and $8(\mathrm{~b})$. We expect that while the density of topologi-

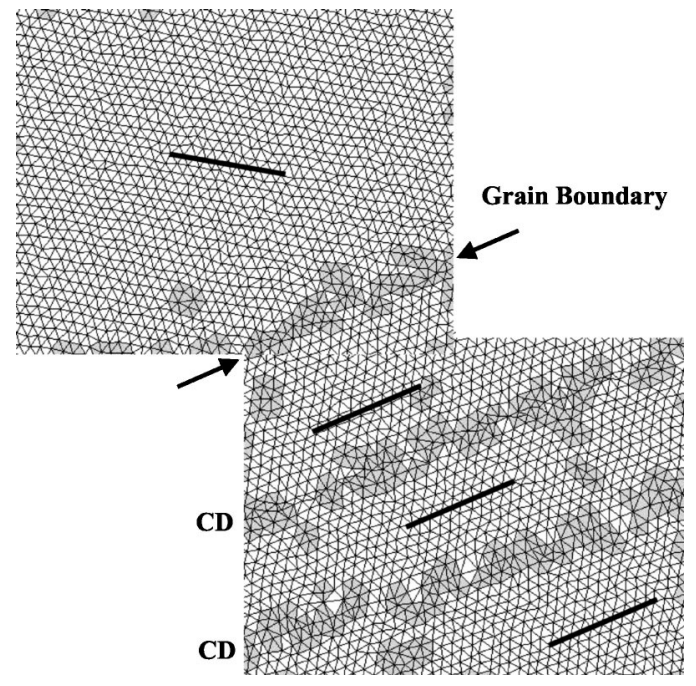

FIG. 7. Delaunay triangulation of the $\mathrm{Bi}_{2} \mathrm{Sr}_{2} \mathrm{CaCl}_{2} \mathrm{O}_{8}$ vortex structure in a FC in 36 Oe down to $4.2 \mathrm{~K}$ depicted in Figs. 6(c) and $6(d)$. The presence of the grain boundary due to the intersection of the two lattice orientations is made evident.

cal defects in a vortex lattice is a characteristic of the vortex structure at the field and temperature where it is frozen, its space distribution is not predetermined. The experiments show that the Fe structure in the Bitter pinning breaks this degeneracy, implying that this pinning should be at least strong enough to reproduce the dislocations at the sites where they were formed. Considering the density of dislocations at $36 \mathrm{Oe}$ (1.2\% of the vortices are located in the dislocation cores) we have estimated that the energy to create the dislocation is equivalent to a pinning energy per vortex of $1 / 400$ of its condensation energy. This is consistent with an
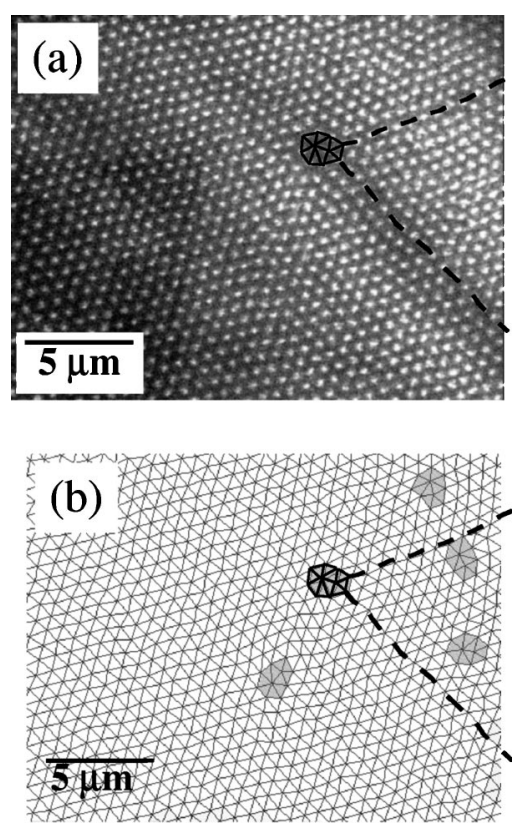

FIG. 8. Bitter pinning in $\mathrm{Bi}_{2} \mathrm{Sr}_{2} \mathrm{CaCu}_{2} \mathrm{O}_{8}$ : Second $\mathrm{FC}$ decoration on top of that shown in Fig. 5. (a) The vortex structure is located on top of the Fe pattern, with no need of dynamical ordering. (b) Corresponding Delaunay triangulation where a dislocation is identified. It is clearly shown that the dislocation present in the Bitter pinning structure is reproduced by the second decoration. 


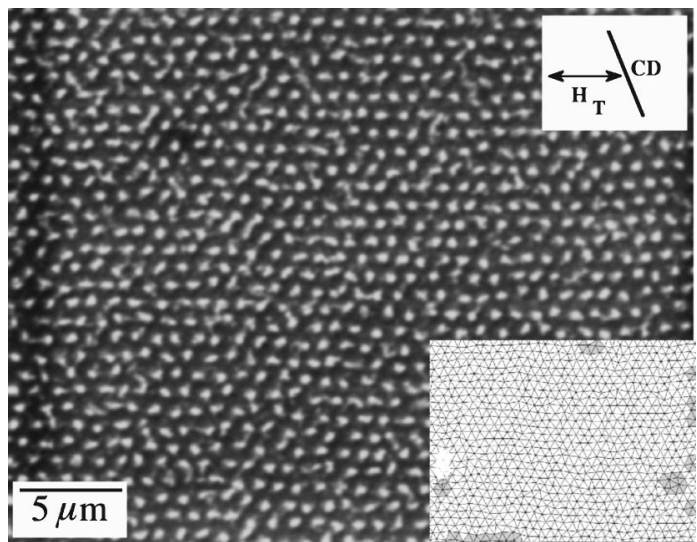

FIG. 9. Vortex positions in a FCR experiment performed at 36 Oe and $4.2 \mathrm{~K}$ in $\mathrm{Bi}_{2} \mathrm{Sr}_{2} \mathrm{CaCu}_{2} \mathrm{O}_{8}$. The picture shows that in FCR experiments the lattice long range orientational order is determined by the direction of the transverse field $H_{T}$. The effect of the rotation overcomes the orientational influence of the correlated defects present in another region of the sample. The correlated defects define the $\mathrm{CD}$ direction. The Delaunay triangulation clearly shows that the principal direction of the unit cell is determined by the transverse field direction; see the inset.

extremely weak pinning of a vortex at a very end of its length, at the surface of the sample.

Since the orientation of the vortex structure when FC is induced by the presence of a correlated potential, we have used the FCR technique to check whether the dynamic memory effect can impose the ordering in a direction different from that given by the defects. The results in Fig. 9 make evident that the FCR experiment at 36 Oe with an angle of $60^{\circ}$ induces long-range orientational order in the defect free region, in a direction parallel to $H_{T}$. This indicates that the bulk force induced by the field rotation overwhelms the one associated with the vortex-vortex interaction at the interface between the crystal and the pinned disordered vortex structure. Despite this, the density of defects in the vortex lattice induced by the rapid field rotation is the same as that in the FC experiment (2\%). Even more, the calculated energy associated to the vortex-vortex interaction in both crystals coincide within $5 \%$. This is a remarkable result indicating that the configuration of the final state is independent of the path followed to create it. In the FC case the vortex structure is frozen at a temperature higher than $4 \mathrm{~K}$, where the bulk pinning sets in, while in the FCR the structure is created by field rotations at $4 \mathrm{~K}$. Note that in the FCR the direction as well as the modulus of the field is changed.

An additional experiment showing that the internal magnetic field follows the evolution of the external one is the following: A FC experiment is made with a magnetic field applied at an angle of $60^{\circ}$ off the $c$ direction and then the transversal component is rapidly suppressed. The vortex structure was found to be crystalline with the six peaks of the Fourier transform located in a circle, the same as that obtained in the FCR case, in contrast with the observed structures in a tilted vortex configuration. ${ }^{25-28}$

Up to now we have discussed experiments where the Bitter pinning is effective in $\mathrm{Bi}_{2} \mathrm{Sr}_{2} \mathrm{CaCu}_{2} \mathrm{O}_{8}$ because the direction of the ordered lattice is determined either by the correlated defects or by the first FCR experiment. A more

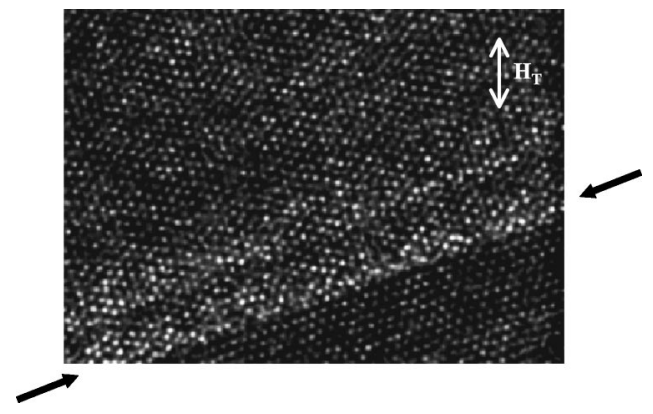

FIG. 10. Magnetic pattern in a Bitter pinning experiment in $\mathrm{Bi}_{2} \mathrm{Sr}_{2} \mathrm{CaCu}_{2} \mathrm{O}_{8}$. The iron pinning pattern is generated by a first magnetic decoration of the vortex lattice in a FC experiment in 36 Oe at $4.2 \mathrm{~K}$ (shown in Fig. 6). The sample is warmed up to room temperature and subsequently a second vortex decoration is made in a FCR experiment in 36 Oe with $60^{\circ}$ of magnetic field rotation at $4.2 \mathrm{~K}$. Despite that the transverse field, $H_{T}$, is not parallel to any of the iron structure principal directions, there is perfect matching of both structures, even in the grain boundary shown in the picture.

stringent proof of the effectiveness of the Bitter pinning is shown in an experiment where the second FCR vortex lattice is oriented in a direction not coincident with that given by any of the lattice vectors of the first decoration. In the particular case shown in Fig. 10 the first decoration was made after $\mathrm{FC}$ in $36 \mathrm{Oe}$, resulting in a magnetic pattern with a compact plane in the direction parallel to that of the correlated defects. The second FCR experiment was performed with $H_{T}$ applied $40^{\circ}$ away from the correlated defects direction. In spite of that, the Bitter pinning locks the new lattice on top of the Fe one.

\section{CONCLUSIONS}

We have proposed a scenario to remark the differences in nucleation and growth processes of vortex structures in low and high temperature superconductors when cooling the sample in the presence of a field. The comparison of experimental results obtained in $\mathrm{NbSe}_{2}$ clean crystals with those in $\mathrm{Bi}_{2} \mathrm{Sr}_{2} \mathrm{CaCu}_{2} \mathrm{O}_{8}$, where the solid structure is formed after a first order transition from the liquid state, support the picture.

The relevance of the weak interaction between replicas inducing perfect matching in macroscopic samples is an unexpected result recently discovered ${ }^{15}$ that makes evident the collective response induced by a small perturbation. This effect cannot be detected by traditional measurements of critical currents (transport or magnetization). We show that the Bitter pinning technique is up to now the only one able to observe in real space how a weak perturbation can break the translational and rotational symmetry in a crystal growth process.

In the case of $\mathrm{NbSe}_{2}$ the Bitter technique makes evident that the Fe clumps do not break the rotational symmetry of the crystal growth in FC experiments. The results show that bulk pinning determines the growth of the grains in the resultant static polycrystalline vortex structure. In this case perfect matching between lattices in the Bitter technique only takes place as a result of the weak interaction between dynamically ordered structures.

In the clean high temperature superconductor $\mathrm{Bi}_{2} \mathrm{Sr}_{2} \mathrm{CaCu}_{2} \mathrm{O}_{8}$ the interaction between the $\mathrm{Fe}$ and vortex 
replica in the second decoration is strong enough to break the translational as well as the rotational symmetry when the static vortex structure is formed. Contrary to what is found in the low temperature superconductor, the results strongly support that the vortex pattern below the solidification temperature corresponds to a long range ordered crystalline structure. The rare grain boundaries found in the decorations are not induced by bulk pinning. They appear as the result of nucleation and growth of crystalline structures in different directions, induced by correlated defects and surface steps. The observed frozen structure is seen to be in or close to the thermodynamic equilibrium state and it is better described by a Bragg glass.

Dynamical ordering and the memory effect at $4 \mathrm{~K}$ (where bulk pinning is strong) applied to $\mathrm{Bi}_{2} \mathrm{Sr}_{2} \mathrm{CaCl}_{2} \mathrm{O}_{8}$ is shown to induce the orientation of the crystalline vortex structure. When the Bitter pinning is applied to a second decoration, with the same field, perfect matching between structures is found. This even when both, the Fe pattern of the first decoration and the second vortex structure are dynamically oriented in different principal crystalline directions. This provides more evidence of the relevance of the weak Fe pinning to break the rotational symmetry of the vortex lattice, when the velocity is quenched from a dynamic state in the presence of strong bulk pinning.

We have shown that when both, the dynamically ordered bitter pinning and the second vortex structure are created by identical magnetic fields, the replicas match independently of the type of material. On the other hand, preliminary results indicate that no matching can be found in the Bitter pinning between commensurate structures with different lattice parameters (different fields). The conditions to maintain matching between structures require that they are formed with the same field intensity (identical replicas). Further research is in progress to elucidate this particular finding.

\section{ACKNOWLEDGMENTS}

The authors would like to acknowledge T. Giamarchi and P. Le Doussal for enlightening discussions. Also they acknowledge C. Winkelmann for discussions of the data and for helping on the energy calculations, E. Kaul for his collaboration in the sample growth, and J. Luzuriaga for a critical reading of the manuscript. This work was partially supported by CONICET (PIP96/4207) and ANPCYT (PICT97/ 03-00061-01116). Y.F. holds a scholarship from FOMEC and M.M. from CONICET; G.N. is a member of CONICET.
${ }^{1}$ G. Blatter M. V. Feigel'man, V. B. Geshkenbein, A. I. Larkin, and V. M. Vinokur, Rev. Mod. Phys. 66, 1125 (1994).

${ }^{2}$ T. Giamarchi and P. Le Doussal, in Statics and Dynamics of Disordered Elastic Systems, edited by A. P. Young (World Scientific, Singapore, 1997), p. 321.

${ }^{3}$ P. Le Doussal and T. Giamarchi, Phys. Rev. B 57, 11356 (1998).

${ }^{4}$ C. Reichhardt, C. Olson, and Franco Nori, Phys. Rev. Lett. 78, 2648 (1997).

${ }^{5}$ K. Harada O. Kamimura, H. Kasai, T. Matsuda, A. Tonomura, and V. V. Moshchalkov, Science 274, 1167 (1996).

${ }^{6}$ J. I. Martin, M. Velez, J. Nogues, and I. K. Schuller, Phys. Rev. Lett. 79, 1929 (1997).

${ }^{7}$ G. J. Dolan and J. H. Dunsmuir, Physica B 152, 7 (1988).

${ }^{8}$ V. V. Moshchalkov M. Baert, V. V. Metlushko, E. Rosseel, M. J. Van Bael, K. Temst, Y. Bruynseraede, and R. Jonckheere, Phys. Rev. B 57, 3615 (1998).

${ }^{9}$ A. E. Koshelev and V. M. Vinokur, Phys. Rev. Lett. 73, 3580 (1994).

${ }^{10}$ U. Yaron P. L. Gammel, D. A. Huse, R. N. Kleiman, C. S. Oglesby, E. Bucher, B. Batlogg, D. J. Bishop, K. Mortensen, K. Claussen, C. A. Bolle, and F. de la Cruz, Phys. Rev. Lett. 73, 2748 (1994).

${ }^{11}$ A. Duarte E. Fernández Righi, C. Bolle, F. de la Cruz, P. L. Gammel, C. S. Oglesby, B. Batlogg, and D. J. Bishop, Phys. Rev. B 53, 11336 (1996).

${ }^{12}$ F. Pardo, F. de la Cruz, P. L. Gammel, C. S. Oglesby, E. Bucher, and D. J. Bishop, Phys. Rev. Lett. 78, 4633 (1997).

${ }^{13}$ F. Pardo, A. Mackenzie, F. de la Cruz, and J. Guimpel, Phys. Rev. B 55, 14610 (1997).

${ }^{14}$ D. J. Bishop et al., in The Vortex State, edited by N. Bontemps, Y. Bruynserade, G. Deutscher, and A. Kapitulnik (Kluwer Academic, Dordrecht, 1994), p. 99.
${ }^{15}$ Yanina Fasano, J. A. Herbsommer, F. de la Cruz, F. Pardo, P. Gammel, E. Bucher, and D. Bishop, Phys. Rev. B 60, R15 047 (1999).

${ }^{16}$ Yanina Fasano, J. A. Herbsommer, and F. de la Cruz, Phys. Status Solidi B 215, 563 (1999).

${ }^{17}$ F. Pardo, F. de la Cruz, P. L. Gammel, E. Bucher, and D. J. Bishop, Nature (London) 396, 348 (1998).

${ }^{18}$ C. Olson, C. Reichhardt, and F. Nori, Phys. Rev. Lett. 81, 3757 (1998).

${ }^{19}$ A. B. Kolton, D. Domínguez, and N. Gronbech-Jensen, Phys. Rev. Lett. 83, 3061 (1999).

${ }^{20}$ F. Pardo, Ph.D. thesis, Instituto Balseiro, Argentina.

${ }^{21}$ E. E. Kaul and G. Nieva, Physica C 341-348, 1342 (2000).

${ }^{22}$ M. Goffman, J. A. Herbsommer, F. de la Cruz, T. W. Li, and P. H. Kes, Phys. Rev. B 57, 3663 (1998).

${ }^{23}$ H. Pastoriza, M. Goffman, A. Arribére, and F. de la Cruz, Phys. Rev. Lett. 72, 2951 (1994); E. Zeldov, D. Majer, M. Konczykowski, V. B. Geshkenbein, V. M. Vinokur, and H. Shtrikman, Nature (London) 375, 373 (1995).

${ }^{24}$ M. R. Koblischka, R. J. Wijngaarden, D. G. De Groot, R. Griessen, A. A. Menovsky, and T. W. Li, Physica C 249, 339 (1995).

${ }^{25}$ C. A. Bolle, F. de la Cruz, P. L. Gammel, J. V. Waszczak, and D. J. Bishop, Phys. Rev. Lett. 71, 4039 (1993).

${ }^{26}$ Y. Paltiel, E. Zeldov, Y. N. Myasoedov, H. Shtrikiman, S. Battacharya, M. J. Higgins, Z. L. Xiao, E. Y. Andrei, P. L. Gammel, and D. J. Bishop, Nature (London) 403, 398 (2000).

${ }^{27}$ Y. Paltiel, E. Zeldov, Y. Myasoedov, M. L. Rappaport, G. Jung, S. Bhattacharya, M. J. Higgins, Z. L. Xiao, E. Y. Andrei, P. L. Gammel, and D. J. Bishop, Phys. Rev. Lett. 85, 3712 (2000).

${ }^{28} \mathrm{~A}$ careful study of the possible existence of the peak effect in these fields is under progress. 